\title{
Un logiciel simple d'utilisation pour évaluer l'incertitude de mesure par la méthode de Monte-Carlo
}

\section{A user-friendly software for the evaluation of measurement uncertainty using a Monte-Carlo method}

\author{
Géraldine EBRARD, Alexandre ALLARD et Nicolas FISCHER
}

Laboratoire national de métrologie et d'essais (LNE), 29 rue Roger Hennequin, 78197 Trappes Cedex, France, Geraldine.Ebrard@lne.fr.

\section{Résumé}

La méthode de Monte-Carlo, décrite dans le Supplément 1 du Guide pour l'expression de l'incertitude de mesure (GUM-S1) est préconisée par les métrologues afin d'évaluer l'incertitude de mesure. Tributaire de ressources informatiques pour la génération de nombres aléatoires, elle demeure plus difficile à mettre en œuvre que la méthode du cumul quadratique décrite dans le GUM, d'où son utilisation parcimonieuse. Dans un but de promouvoir la méthode de Monte-Carlo au sein de la communauté métrologique, le Laboratoire national de métrologie et d'essais a développé un logiciel nommé «LNE-MCM». Facile à prendre en main, il permet d'implémenter la méthode de propagation des distributions par simulations de Monte-Carlo décrite dans le GUM-S1. Allant au-delà du GUM-S1, le logiciel LNE-MCM propose à l'utilisateur de réaliser une analyse de sensibilité, de tester l'adéquation de sa distribution de sortie à des lois théoriques et de traiter le cas de mesurandes multiples. Le logiciel LNE-MCM a été mis à disposition gratuitement sur le site internet du LNE en janvier 2016.

MOTS CLÉS : INCERTITUDE DE MESURE, MÉTHODE DE MONTE-CARLO, SUPPLÉMENT 1 DU GUM, ANALYSE DE SENSIBILITÉ.

\footnotetext{
Abstract

The Monte-Carlo Method, described in the Guide for Uncertainty Measurement Supplement 1, is advocated by metrologists to evaluate the uncertainty of measurement. This method requires computational resources to generate random numbers, which is a limit for a common use compared with the GUM uncertainty framework. In order to popularize the Monte-Carlo method, LNE-MCM software was developed as a user-friendly software by the Laboratoire national de métrologie et d'essais. LNE-MCM is dedicated to the evaluation of measurement un-
}

certainty using Monte Carlo simulations according to the principles of the supplement 1 to the GUM. Moreover, additional features are implemented: the case of multivariate models, sensitivity analysis to provide an uncertainty budget and a goodness-of-fit test for the samples of the output quantities. LNE-MCM is freely available on LNE's website since January 2016

KEYWORDS: MEASUREMENT UNCERTAINTY, MONTE-CARLO METHOD, SUPPLEMENT 1 TO THE GUM, SENSITIVITY ANALYSIS.

\section{Introduction}

La méthode de référence utilisée pour évaluer une incertitude de mesure est décrite dans le Guide pour l'expression de l'incertitude de mesure (GUM) [1]. Cette méthode repose sur le principe de la propagation des variances. Largement utilisée du fait de sa mise en œuvre simple, les conditions d'application de la méthode décrite dans le GUM ne sont pas toujours remplies. À titre d'exemple, lorsque le modèle est fortement non linéaire, l'approximation linéaire correspondant à l'utilisation de la formule de propagation du GUM à l'ordre 1 ne permet pas de fournir une estimation satisfaisante de la variance de la grandeur de sortie [2]. Si le GUM autorise à effectuer un développement de Taylor à l'ordre 2, cela induit des calculs supplémentaires potentiellement compliqués. Dans une telle situation, le GUM-S1 préconise d'utiliser la méthode qui repose sur le principe de propagation de distributions en utilisant des simulations 


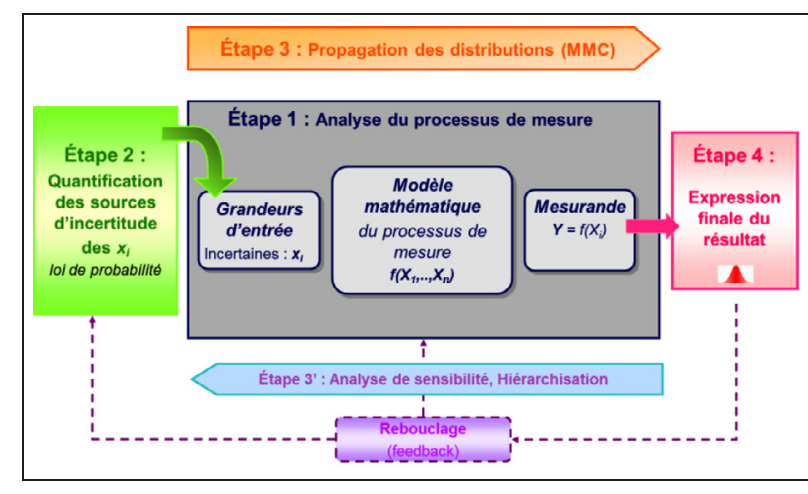

Fig. 1. - Découpage en quatre étapes de la méthode de MonteCarlo.

de type Monte-Carlo. Cette méthode, communément appelée «méthode de Monte-Carlo», est décrite dans le GUM-S1 [3]. C'est une procédure stochastique consistant à tirer au sort des valeurs dans une loi de probabilité assignée à une grandeur au moyen d'un générateur de nombres pseudo-aléatoires. Elle est, par conséquent, tributaire d'outils informatiques pour sa mise en œuvre. C'est dans ce contexte que l'application LNE-MCM [4] a été développée avec le logiciel MATLAB. Cette application possède un générateur de nombres pseudo-aléatoires ayant de bonnes qualités statistiques et fondé sur les travaux de Mersenne Twister's [5]. Il est cité dans le GUM-S1 [3, § 9.1.4] et dans la norme ISO 28640 [6], qui spécifie les méthodes pouvant être employées pour générer des variables aléatoires uniformes et non-uniformes en vue d'effectuer des simulations de Monte-Carlo. L'interface conviviale aiguille l'utilisateur en rappelant le découpage en quatre étapes de la méthode de MonteCarlo (fig. 1). En amont de l'utilisation du logiciel LNEMCM, le processus de mesure doit être établi. En d'autres termes, l'utilisateur doit connaître le modèle de mesure explicite et les lois de probabilité des grandeurs d'entrée. Pour affecter une distribution aux grandeurs d'entrée, quatorze lois sont disponibles : bêta, curvilinéaire trapézoïdale, dérivée d'arc sinus, exponentielle, gamma, log-normale, normale, normale tronquée, normale tronquée inférieure, normale tronquée supérieure, Poisson, Student, triangulaire, uniforme. Conformément au GUM$\mathrm{S} 1$, le logiciel LNE-MCM autorise les corrélations entre les grandeurs d'entrée modélisées par des lois normales, on parle dans ce cas, de distributions gaussiennes multivariées. Dans le logiciel LNE-MCM, les grandeurs d'entrée observées simultanément peuvent également être modélisées à partir de distributions de Student. Dans ce dernier cas, la dépendance est modélisée à l'aide d'une copule gaussienne avec des marginales Student [7]. Afin de vérifier la validité des résultats obtenus avec la méthode du GUM, la procédure de validation décrite dans le GUM-S1 a été mise en œuvre. Dédié initialement à l'estimation de l'incertitude de mesure par la méthode de Monte-Carlo, le logiciel LNE-MCM a été étendu à certains cas de mesurandes multiples traités dans le Supplément 2 du GUM [8]. De plus, il offre une analyse de sensibilité par le biais de deux méthodes : le coefficient de corrélation des rangs de Spearman pour les modèles monotones et les indices de Sobol' pour des modèles plus complexes $[9,10]$. Enfin, il permet de déterminer la loi de la grandeur de sortie en vérifiant avec le test de Kolmogorov-Smirnov [11] l'adéquation à différentes lois théoriques (normale, Student, log-normale, gamma, bêta, uniforme, exponentielle). L'illustration de cet outil se fera au moyen de deux exemples décrits dans les deux référentiels cités précédemment, en l'occurrence les Suppléments 1 et 2 du GUM.

\section{Méthode de Monte-Carlo}

\subsection{GUM $v s$. GUM-S1}

La méthode de Monte-Carlo est une méthode alternative à la méthode décrite dans le guide pour l'expression de l'incertitude de mesure (GUM) lorsque les conditions d'applicabilité de la méthode GUM ne sont pas remplies [3]. En effet, dans le GUM, l'évaluation de l'incertitude de mesure est généralement basée sur un développement de Taylor du premier ordre, ce qui est problématique dans le cas de modèles fortement non linéaires. De plus, lorsque la distribution de la grandeur de sortie $Y$ s'écarte sensiblement d'une distribution gaussienne, l'utilisation d'un facteur d'élargissement $k=2$ ne permet plus d'aboutir à un intervalle élargi contenant environ $95 \%$ des valeurs que l'on peut attribuer au mesurande. La méthode de Monte-Carlo, quant à elle, fournit deux types d'intervalles élargis, qui ne nécessitent pas $\mathrm{d}$ 'hypothèse sur la distribution de la grandeur de sortie, en l'occurrence l'intervalle symétrique en probabilité et l'intervalle le plus court. On rappelle qu'un intervalle élargi $\left[y_{\text {inf }} ; y_{\text {sup }}\right]$ de probabilité $p(0 \leq p \leq 1)$ est un intervalle qui contient $(100 \times p) \%$ des valeurs attribuées à $Y$ issues de la simulation de Monte-Carlo. L'intervalle symétrique en probabilité est obtenu en prenant pour $y_{\text {inf }}$ le quantile d'ordre $(1-p) / 2$ et pour $y_{\text {sup }}$ le quantile d'ordre $(1+p) / 2$. L'intervalle le plus court est obtenu en déterminant $\alpha(0 \leq \alpha \leq 1-p)$ tel que l'intervalle constitué par les quantiles d'ordres $\alpha$ et $(p+\alpha)$ soit le plus court possible. Dans le cas d'une distribution de probabilité symétrique, ces deux intervalles sont identiques.

\subsection{Découpage en quatre étapes}

La méthode de Monte-Carlo, comme la méthode GUM, peut se résumer en quatre grandes étapes (fig. 1).

Dans la première étape, l'analyse du processus de mesure permet de définir le mesurande, de recenser les grandeurs d'entrée et de déterminer le modèle de mesure. Puis, grâce à l'information disponible (tolérance, certificat d'étalonnage, mesures répétées, avis d'experts...), des densités de probabilité sont assignées aux grandeurs d'entrée. Dans la troisième étape, les distributions sont propagées, par simulation de Monte-Carlo, à travers le modèle mathématique. In fine, les résultats sont résumés au moyen de paramètres statistiques tels que la moyenne, l'écart type et les intervalles élargis. Le logiciel LNE-MCM est un outil de calcul, pas de modélisation. Il ne permet pas à l'utilisateur d'établir le modèle 
mathématique du processus de mesure, ni de déterminer les grandeurs d'entrée et leurs éventuelles corrélations. Le logiciel LNE-MCM est utilisable lorsque l'analyse du processus est réalisée.

\subsection{Corrélation des grandeurs d'entrée}

Le GUM-S1 se limite à des corrélations entre les grandeurs d'entrée modélisées par des lois normales. Cependant en pratique, il peut y avoir des corrélations entre différentes séries de mesures. Si celles-ci sont modélisées par une loi de Student, le logiciel LNE-MCM permet également de traiter cette corrélation.

Dans le cas de $q$ grandeurs d'entrée $\mathbf{X}=\left(X_{1}, \ldots\right.$, $\left.X_{q}\right)^{T}$ modélisées chacune par une loi normale, une distribution gaussienne multivariée est assignée à $\mathbf{X}$. La densité de probabilité jointe correspondante est donnée, pour tout $\xi$ appartenant à $\mathbb{R}^{q}$, par la formulation suivante

$$
g_{\mathbf{X}}(\boldsymbol{\xi})=\frac{1}{\left[(2 \pi)^{q} \operatorname{det} \mathbf{U}_{\mathbf{X}}\right]^{0,5}} \exp \left(-\frac{1}{2}(\boldsymbol{\xi}-\mathbf{x})^{T} \mathbf{U}_{\mathbf{X}}^{-1}(\boldsymbol{\xi}-\mathbf{x})\right),
$$

où $\mathbf{x}$ représente le vecteur formé des moyennes des $q$ grandeurs d'entrée de lois normales $(E(\mathbf{X})=\mathbf{x})$ et $\mathbf{U}_{\mathbf{X}}$ est la matrice de variance-covariance associée à $\mathbf{X}$ $\left(V(\mathbf{X})=\mathbf{U}_{\mathbf{X}}\right)$.

Dans le cas de lois de Student, il s'agit de prendre en compte la corrélation entre des grandeurs d'entrée dont la loi marginale est une loi de Student. Ceci a été effectué en utilisant des copules gaussiennes selon la procédure décrite par Possolo [7]. Ce choix de modélisation est particulièrement approprié au cas de grandeurs d'entrée observées simultanément pour lesquelles seul un nombre limité d'observations est disponible.

\section{Validation des résultats obtenus selon le GUM par la méthode de Monte-Carlo}

Bien qu'ayant un domaine de validité plus étroit que celui relatif à la méthode de Monte-Carlo, la méthode décrite dans le GUM est largement répandue du fait de sa mise en place simple et de ses résultats immédiats. Se pose alors la question de la validité de ses résultats. Afin de répondre à cette problématique, le GUM-S1 a défini un critère de validation fondé sur la comparaison des intervalles élargis obtenus par les deux méthodes. En pratique, on définit la tolérance numérique $\delta=0,5 \times 10^{l}$ avec $l$ un entier tel que l'écart type sur les valeurs attribuées à la grandeur de sortie obtenues par Monte-Carlo s'écrive $u(y)=c \times 10^{-l}$ où $c$ est un entier à $n_{\text {dig }}$ chiffres $\left(n_{\text {dig }}=1\right.$ ou 2 selon que l'on valide la méthode GUM à 1 ou 2 chiffres significatifs $[3, \S 8.1])$. On note $U_{p}=k_{p} \times u(y)$ l'incertitude élargie et $\left[y-U_{p} ; y+U_{p}\right]$ l'intervalle à $100 p \%$ obtenus par la méthode du GUM. De même on note $y_{\text {inf }}$ et $y_{\text {sup }}$ les bornes de l'intervalle élargi à $100 p \%$ le plus court obtenu par simulations de Monte-Carlo. Soient $d_{\text {inf }}$ et $d_{\text {sup }}$, les différences absolues entre les bornes inférieures et supérieures des deux intervalles, données par:

$$
d_{\text {inf }}=\left|y-U_{p}-y_{\text {inf }}\right| \quad \text { et } \quad d_{\text {sup }}=\left|y+U_{p}-y_{\text {sup }}\right| .
$$

$\operatorname{Si} \inf \left(d_{\text {inf }}, d_{\text {sup }}\right) \leq \delta$ alors les résultats obtenus par la méthode GUM sont comparables aux résultats obtenus par Monte-Carlo. Les hypothèses de la méthode GUM sont alors validées. Dans le cas contraire, il convient de s'affranchir de la méthode de propagation des variances au profit de la méthode de Monte-Carlo.

Afin de répondre à des besoins allant au-delà de la simple application du GUM-S1, des fonctionnalités supplémentaires ont été implémentées dans le logiciel LNE-MCM.

\section{Fonctionnalités avancées pour le calcul d'incertitude}

\subsection{Mesurande multiple}

Le GUM-S1 est dédié à la méthode de Monte-Carlo pour un mesurande unique. En 2011, le JCGM (Joint Committee for Guides in Metrology) a publié le Supplément 2 du GUM [8] permettant d'étendre à la fois la formule de propagation des variances et celle des distributions au cas de mesurandes multiples ou d'un nombre quelconque de grandeurs de sortie. Dans la réalité, de nombreux processus de mesure portent sur des mesurandes multiples. On peut citer, par exemple, le domaine de la chimie pour lequel les mesurandes peuvent être la concentration de différents éléments présents en solution, ou le domaine de l'électricité pour lequel le mesurande est souvent décomposé en module et phase. Lorsque des sources d'incertitude sont communes à plusieurs mesurandes, celles-ci entraînent des covariances entre les estimations des mesurandes. Si ceux-ci sont ensuite utilisés au sein d'un nouveau modèle de mesure, il est primordial de tenir compte des corrélations existantes entre leurs estimations. En effet, traiter de façon indépendante des mesurandes corrélés ne permet pas de rendre compte du comportement réel des grandeurs. Au contraire, la détermination d'une région élargie en lieu et place de la juxtaposition d'intervalles élargis individuels permet une représentation de la connaissance des mesurandes plus fidèle à la réalité. Conformément à la définition du Supplément 2 du GUM, une région élargie, $R_{Y}$, attribuée au mesurande vectoriel $\mathbf{Y}=\left(Y_{1}, \ldots, Y_{m}\right)^{T}$ est un espace de dimension $m$ tel que la probabilité que $\mathbf{Y}$ réside dans $R_{Y}$ soit égale à la probabilité de couverture stipulée $p$. Il existe un nombre arbitraire de régions élargies en analyse multivariée. Trois cas particuliers de régions élargies sont introduits dans le Supplément 2 du GUM : l'ellipse multivariée, le rectangle multivarié et la plus petite région de confiance. Dans le logiciel LNE-MCM, seule la représentation ellipsoïdale dans le plan (dimension 2) a été implémentée. Pour ce faire, on représente les couples de mesurandes. Sont notés y le vecteur des moyennes de chaque mesurande, $\mathbf{U}_{\mathbf{y}}$ la matrice de variance-covariance et $k_{p}$ le quantile d'ordre $p$. L'équation de l'ellipse vérifie pour tout $\boldsymbol{\eta} \in R^{2}$,

$$
(\boldsymbol{\eta}-\mathbf{y})^{T} \mathbf{U}_{\mathbf{y}}^{-1}(\boldsymbol{\eta}-\mathbf{y})=k_{p}^{2}
$$

où $\mathbf{y}$ représente le centre de l'ellipse, $\mathbf{U}_{\mathbf{y}}$ sa forme et $k_{p}$ sa taille. Le quantile $k_{p}$ est une constante déterminée de telle 
sorte que l'aire sous la fonction de densité de probabilité multidimensionnelle sur la région elliptique soit égale à $p$ $(0 \leq p \leq 1)$. Notons que la représentation ellipsoïdale de la région élargie correspond à la région élargie de plus petite surface lorsque $\mathbf{Y}$ suit approximativement une loi normale bivariée.

Les grandeurs $\mathbf{y}, \mathbf{U}_{\mathbf{y}}$ et $k_{p}$ sont évaluées à partir des valeurs des mesurandes simulées par la méthode de Monte-Carlo.

Le logiciel LNE-MCM permet ainsi de visualiser les mesurandes deux à deux et de calculer la covariance (ou le coefficient de corrélation linéaire) associée à leur estimation.

\subsection{Analyse de sensibilité}

Pour le métrologue, le budget d'incertitude final constitue un outil important pour l'analyse de son processus de mesure. En effet, il permet de connaître les contributions des différentes sources d'incertitude à l'incertitude finale et d'identifier immédiatement quelles sont les sources d'incertitude prépondérantes, ou à l'inverse, quelles sont celles qui sont peu influentes et qui pourraient être considérées comme des constantes dans le modèle mathématique du processus de mesure.

\subsubsection{Méthode décrite dans le Supplément 1 du GUM}

En appliquant la formule de propagation des variances, le budget d'incertitude s'obtient facilement et directement par l'intermédiaire du calcul des dérivées partielles, à condition que le modèle mathématique ait une expression explicite. En effet, les composantes d'incertitude attribuées aux $N$ grandeurs d'entrée s'écrivent $u_{i}(y)=\left|c_{i}\right| u\left(x_{i}\right)$ avec $c_{i}$ le $i^{e}$ coefficient de sensibilité, correspondant à la dérivée partielle de $y$ par rapport à $x_{i}$, et $u\left(x_{i}\right)$ l'incertitude type associée à la grandeur $x_{i}(i=$ $1, \ldots, N)$. Par contre, ces coefficients de sensibilité ne sont pas calculés dans le cas de la propagation des distributions par simulation de Monte-Carlo. Le GUM-S1 propose alors de faire varier les grandeurs d'entrée chacune à leur tour (en fixant les autres à leur meilleure estimation), puis de comparer l'incertitude type obtenue $\tilde{u}_{i}(y)$ à l'incertitude type $u(y)$ obtenue en faisant varier toutes les grandeurs d'entrée. Cette méthode de sensibilité locale présente deux inconvénients. D'une part elle nécessite des calculs supplémentaires (autant de simulations à effectuer que de grandeurs d'entrée dans le modèle de mesure) et d'autre part elle ne permet pas de quantifier l'effet d'éventuelles interactions entre les grandeurs d'entrée si le modèle de mesure n'est pas linéaire.

L'établissement d'un budget d'incertitude est connu dans la littérature sous l'appellation « analyse de sensibilité ». Différentes méthodes existent pour effectuer une analyse de sensibilité et déterminer un budget d'incertitude [10]. Deux méthodes de sensibilité globale ont été retenues et mises en œuvre dans le logiciel LNE-MCM : le coefficient de corrélation de Spearman et les indices de Sobol' [12].

\subsubsection{Coefficient de corrélation de Spearman}

Le coefficient de corrélation de Spearman [12] permet de donner une mesure de l'intensité de la relation entre deux variables quantitatives et également son sens lorsque cette relation est monotone qu'elle soit linéaire ou pas. Le calcul ne porte pas sur les valeurs de ces variables, issues des simulations de Monte-Carlo effectuées pour propager les distributions, mais sur leurs rangs attribués suite au classement des valeurs par ordre croissant. Le coefficient de corrélation de Spearman entre une grandeur d'entrée $X_{i}$ et une grandeur de sortie $Y_{k}$ s'obtient alors comme :

$$
\rho_{X_{i} ; Y_{k}}=1-\frac{6 \sum_{j=1}^{M}\left(r_{i, j}-r_{k, j}\right)^{2}}{M\left(M^{2}-1\right)},
$$

où $r_{i, j}$ et $r_{k, j}$ représentent le rang de la $j^{e}$ itération (parmi les $M$ tirages de Monte-Carlo) des grandeurs $X_{i}$ et $Y_{k}$ respectivement.

Afin d'obtenir une expression de la contribution de la grandeur d'entrée $X_{i}$ vis-à-vis de la grandeur de sortie $Y_{k}$ et ceci pour les $N$ grandeurs d'entrée, ce coefficient de corrélation est normalisé selon :

$$
S_{X_{i} ; Y_{k}}^{\text {Spear }}=\frac{\rho_{X_{i} ; Y_{k}}^{2}}{\sum_{l=1}^{N} \rho_{X_{l} ; Y_{k}}^{2}} .
$$

L'avantage de cette méthode, en dehors de sa simplicité de mise en œuvre, est qu'elle ne nécessite pas de simulations supplémentaires.

\subsubsection{Indices de Sobol'}

Pour des modèles plus complexes, pour lesquels des interactions entre les grandeurs d'entrée peuvent être significatives, le logiciel LNE-MCM permet d'estimer des indices de Sobol' [9]. Ces indices de sensibilité reposent sur la décomposition de la variance de la grandeur de sortie $Y$ :

$$
V(Y)=V\left(E\left(Y \mid X_{i}\right)\right)+E\left(V\left(Y \mid X_{i}\right)\right) .
$$

Dans cette décomposition, le premier terme, $V_{i}=$ $V\left(E\left(Y \mid X_{i}\right)\right)$ représente la part de variance de la grandeur de sortie qui est due aux variations de la grandeur d'entrée $X_{i}$, alors que le second terme, $E\left(V\left(Y \mid X_{i}\right)\right)$, représente la part de variance de la grandeur de sortie due aux variations des autres grandeurs d'entrée (aussi appelée variance résiduelle). Ainsi, la contribution de la variable $X_{i}$ seule à la variance de la grandeur de sortie est donnée par :

$$
S_{i}=\frac{V\left(E\left(Y \mid X_{i}\right)\right)}{V(Y)}=\frac{V_{i}}{V(Y)} .
$$

En considérant la variance de l'espérance conditionnelle par rapport à deux variables $X_{i}$ et $X_{j}$, on peut 
également en déduire la contribution $S_{i j}$ à la variance de la grandeur de sortie de l'interaction entre $X_{i}$ et $X_{j}$ :

$$
S_{i j}=\frac{V\left(E\left(Y \mid X_{i}, X_{j}\right)\right)-V_{i}-V_{j}}{V(Y)}=\frac{V_{i j}-V_{i}-V_{j}}{V(Y)} .
$$

L'estimation de ces indices de Sobol' par la méthode de Monte-Carlo nécessite de réaliser des simulations supplémentaires. Cela peut être très coûteux en temps de calcul selon la complexité du modèle de mesure. L'avantage de cette méthode est qu'elle permet d'obtenir une décomposition exhaustive de la variance de la grandeur de sortie, prenant en compte les éventuelles interactions d'ordre 2 intervenant dans le modèle de mesure.

\subsection{Test d'adéquation}

La propagation des distributions par la méthode de Monte-Carlo fournit en sortie la distribution de probabilité empirique associée à la grandeur de sortie. Dans le cas où celle-ci s'avère être une grandeur d'entrée pour un autre modèle de mesure, il est important de pouvoir lui attribuer une distribution de probabilité. Pour cela, le logiciel LNE-MCM permet de déterminer la loi de la grandeur de sortie ou la loi la plus probable en vérifiant, avec le test de Kolmogorov-Smirnov [11], l'adéquation à différentes lois théoriques, en l'occurrence les lois normale, uniforme, exponentielle, log-normale, gamma, bêta et Student. Le principe du test de Kolmogorov-Smirnov est de comparer la fonction de répartition simulée de la grandeur de sortie, $F_{M}$, définie par:

$$
F_{M}(y)=\frac{1}{M} \sum_{j=1}^{M} 1_{\left\{Y_{j} \leq y\right\}}
$$

avec la fonction de répartition $F_{0}$ d'une loi théorique. La grandeur $M$ représente le nombre de tirages de MonteCarlo et $Y_{j}$ représente la $j^{e}$ valeur de l'échantillon simulé. Les paramètres de la loi théorique sont estimés à partir des observations $Y_{j, j=1, \ldots, M}$ de la grandeur de sortie par le principe du maximum de vraisemblance. La statistique du test de Kolmogorov-Smirnov est donnée par la formule suivante :

$$
D_{M}=\max _{1 \leq i \leq M}\left\{\left|F_{0}\left(Y_{i}\right)-F_{M}\left(Y_{i}\right)\right|\right\} .
$$

Si les différences entre les deux fonctions de répartition sont statistiquement significatives, alors le test conduit à la conclusion que la grandeur de sortie ne suit pas la loi théorique considérée au niveau de risque $\alpha$ spécifié. Par défaut, $\alpha$ est égal à 0,05 . Le logiciel LNE-MCM donne les valeurs de la statistique du test et de la valeur $p$ pour chaque loi théorique. La valeur $p$ est la probabilité d'obtenir une valeur de la statistique de test supérieure ou égale à $D_{M}$ sous l'hypothèse que les valeurs simulées sont distribuées selon la loi théorique. En d'autres termes, plus cette valeur est petite, plus les écarts entre la loi de la grandeur de sortie et la loi théorique sont grands. Les résultats sont résumés dans un tableau accompagné d'un graphique représentant la densité de probabilité de la grandeur de sortie calculée et les densités des sept lois de probabilité théoriques. Un classement par ordre décroissant en fonction de la valeur $p$ est réalisé. La pratique courante est de rejeter la loi théorique si la valeur $p$ est inférieure à 0,05 . Dans le cadre d'une simulation de Monte-Carlo avec un grand nombre de tirages, le test est très sensible à d'infimes déviations à la loi théorique et les valeurs $p$ sont très majoritairement faibles. De ce fait, il convient également de s'intéresser à la valeur de la statistique du test puisque plus celle-ci est faible, plus la loi théorique est proche de celle de la grandeur de sortie. Si la fonction de répartition simulée ne correspond à aucune des fonctions de répartition des lois théoriques proposées, il convient de choisir la loi théorique qui s'approche le plus de l'échantillon testé.

\section{Exemples}

Les fonctionnalités du logiciel LNE-MCM sont illustrées à partir de deux exemples traités dans deux référentiels cités dans cet article, en l'occurrence le GUM-S1, traitant de l'étalonnage de masse, et le Supplément 2 du GUM, traitant de la relation entre le système de coordonnées cartésiennes et le système de coordonnées polaires.

\section{1. Étalonnage de masse (GUM-S1)}

À titre d'illustration, nous reprenons le modèle mathématique décrit dans le GUM-S1 [3, § 9.3] et les distributions attribuées aux grandeurs d'entrée. Le mesurande $d m$ [exprimé en $\mathrm{kg}$ ], correspondant à l'écart de la masse conventionnelle à la valeur nominale $m_{\text {nom }}$, s'exprime en fonction de six grandeurs d'entrée par la formulation suivante :

$$
d m=\left(m_{R, c}+\delta m_{R, c}\right)\left[1+\left(\rho_{a}-\rho_{a_{0}}\right)\left(\frac{1}{\rho_{W}}-\frac{1}{\rho_{R}}\right)\right]-m_{n o m},
$$

avec :

$$
\begin{aligned}
& m_{R, c} \sim N\left(0,1 ; 5 \times 10^{-8}\right)(\mathrm{kg}), \\
& \delta m_{R, c} \sim N\left(1,234 \times 10^{-6} ; 2 \times 10^{-8}\right)(\mathrm{kg}), \\
& \rho_{a} \sim U(1,1 ; 1,3)\left(\mathrm{kg} \cdot \mathrm{m}^{-3}\right), \\
& \rho_{W} \sim U(7000 ; 9000)\left(\mathrm{kg} \cdot \mathrm{m}^{-3}\right), \\
& \rho_{R} \sim U(7950 ; 8050)\left(\mathrm{kg} \cdot \mathrm{m}^{-3}\right), \\
& m_{\text {nom }}=0,1 \mathrm{~kg}, \\
& \rho_{a_{0}}=1,2 \mathrm{~kg} \cdot \mathrm{m}^{-3} .
\end{aligned}
$$

Le découpage en quatre étapes du calcul par MonteCarlo est facilement identifiable (fig. 2 et 3 ).

L'étape 4 (fig. 3) renseigne sur la moyenne et l'écart type des valeurs du mesurande simulées, avec un nombre de simulations $M=10^{6}$. En outre, l'utilisateur peut paramétrer l'intervalle élargi en choisissant la probabilité de couverture et en privilégiant l'intervalle le plus court ou symétrique en probabilité. Le graphique représentant la densité de probabilité empirique ainsi que les bornes de l'intervalle choisi permet à l'utilisateur de visualiser 


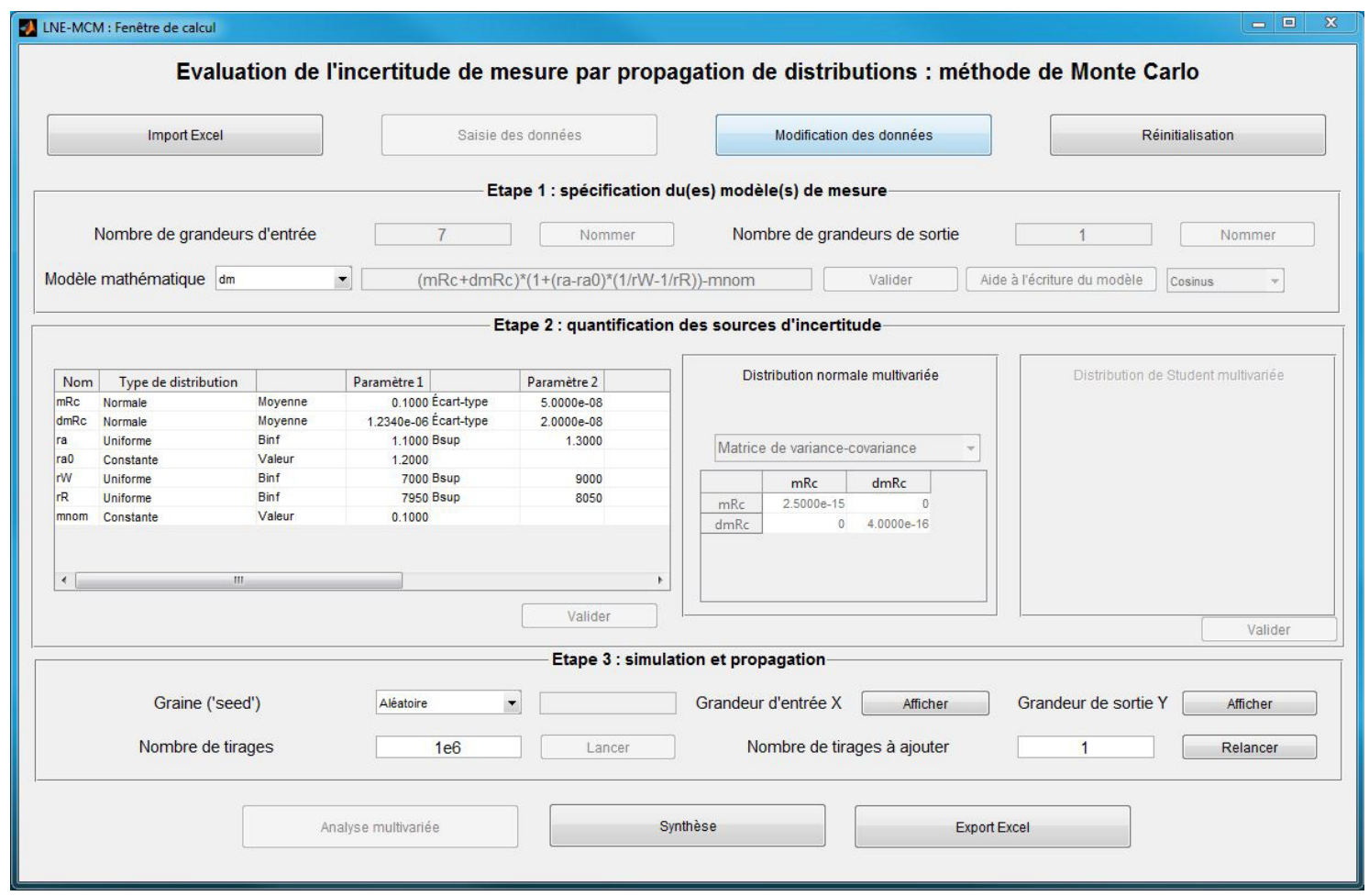

Fig. 2. - Fenêtre de calcul comprenant les trois premières étapes du processus de calcul.



Fig. 3. - Fenêtre comprenant l'étape 4 du processus de calcul et donnant accès aux fonctionnalités avancées.

la distribution du mesurande et de se positionner par rapport à une évaluation faite par la méthode décrite dans le GUM. Ces résultats sont comparés à ceux obtenus dans le GUM-S1 (tab. 1).

Pour vérifier la validité de l'évaluation faite selon le GUM le cas échéant, le logiciel LNE-MCM met en œuvre la procédure de validation décrite dans le GUM-S1. Ayant réalisé son calcul par la méthode de propagation des variances, l'utilisateur saisit la valeur attribuée
Tableau 1

Résultats obtenus selon GUM-S1 et le logiciel LNE-MCM.

\begin{tabular}{|c|c|c|}
\hline & GUM-S1 & LNE-MCM \\
\hline $\begin{array}{c}\text { Valeur attribuée } \\
\text { au mesurande } y\end{array}$ & 1,234 & 1,234 \\
\hline $\begin{array}{c}\text { Incertitude type } \\
u(y)\end{array}$ & 0,075 & 0,075 \\
\hline $\begin{array}{c}\text { Intervalle } \\
\text { élargi }\end{array}$ & {$[1,083 ; 1,383]$} & {$[1,083 ; 1,383]$} \\
\hline
\end{tabular}




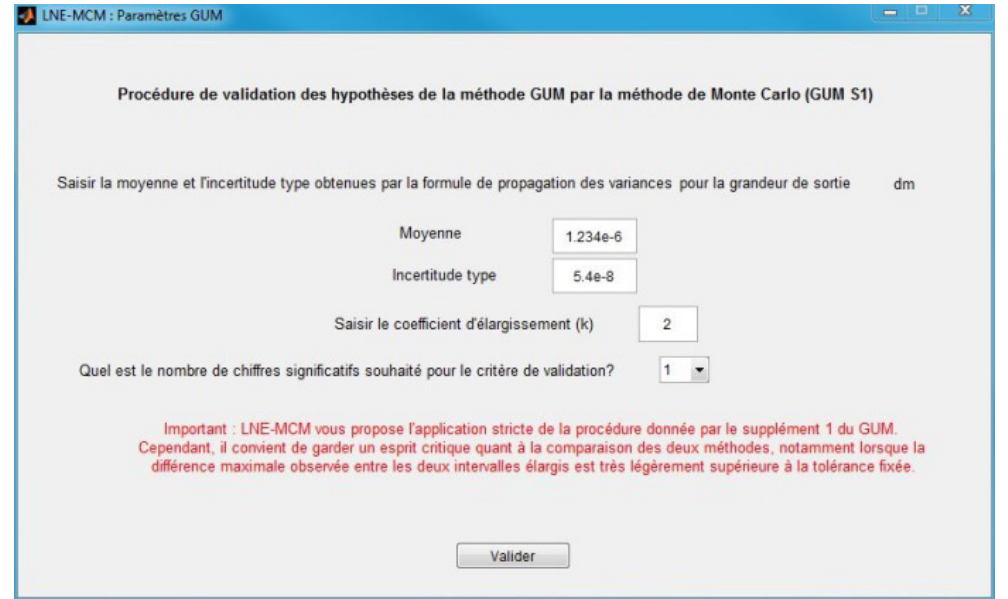

Fig. 4. - Fenêtre de saisie pour la validation des résultats du GUM par Monte-Carlo.

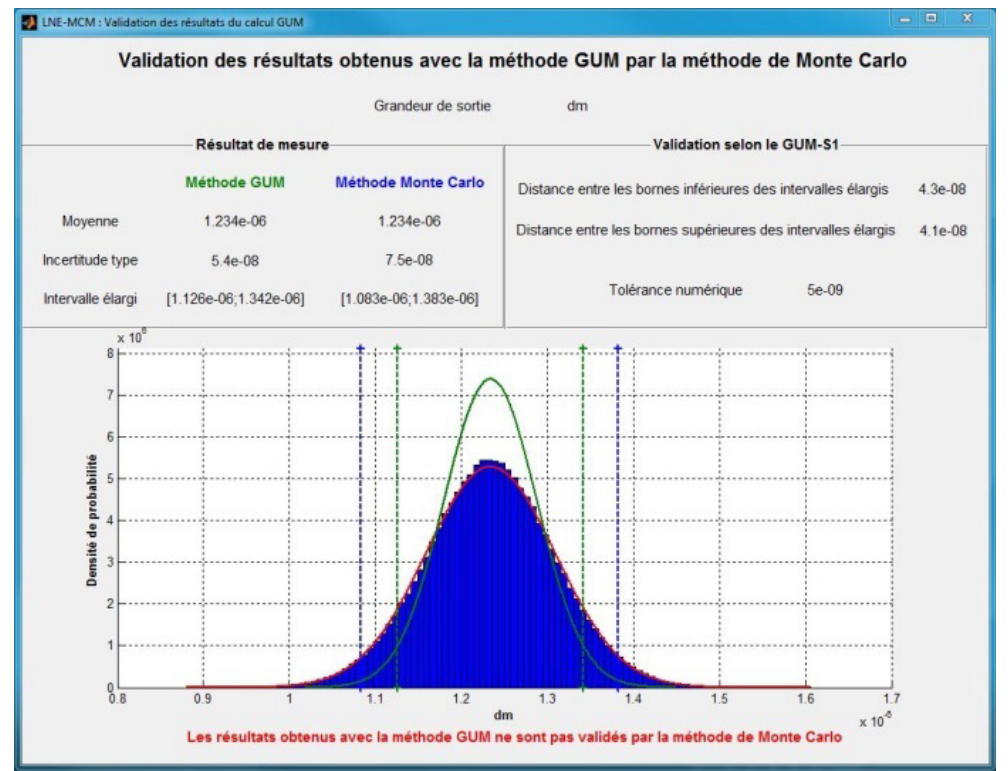

Fig. 5. - Procédure de validation des résultats GUM selon le critère du GUM-S1.

au mesurande (moyenne), l'incertitude type (écart type) ainsi que le facteur d'élargissement utilisé et le degré de précision de sa validation (1 ou 2 chiffres significatifs) comme indiqué dans la figure 4.

Les résultats de mesure obtenus par les deux méthodes sont résumés dans un cadre dédié et une phrase de conclusion relative à la validation des résultats obtenus selon le GUM oriente l'utilisateur quant à son choix de méthode d'évaluation de l'incertitude (fig. 5). Dans cet exemple, la méthode GUM (avec uniquement les termes d'ordre 1 dans la formule de propagation de l'incertitude) fournit une incertitude type éloignée de celle donnée par la méthode de Monte-Carlo. En effet, pour ce modèle, il est nécessaire de prendre en compte les termes d'ordre 2 en raison de l'interaction entre les deux masses volumiques $\rho_{a}$ et $\rho_{W}$. Ainsi, en cas de doute, l'application des deux méthodes peut conduire à remettre en cause la méthode GUM à l'ordre 1 et à évaluer l'incertitude soit en prenant en compte les termes d'ordre 2, soit en utilisant le résultat de la méthode de Monte-Carlo.

Afin de déterminer la loi de probabilité associée à la grandeur de sortie $d m$, le test d'adéquation de Kolmogorov-Smirnov est réalisé (fig. 6).

Ainsi, on constate que le test de KolmogorovSmirnov rejette toutes les lois théoriques considérées (valeur $p<0,05$ ) au seuil $5 \%$. Cependant, la loi normale est la plus proche de la loi empirique. Par conséquent, compte tenu également de la forme de la densité de probabilité, le choix d'une loi normale peut constituer une approximation acceptable pour représenter la grandeur de sortie. Concernant les grandeurs d'entrée, il est également intéressant de connaître le budget d'incertitude. Dans cet exemple, la méthode d'analyse de sensibilité privilégiée est celle qui repose sur le calcul des indices de Sobol'. La représentation sous forme de camembert 


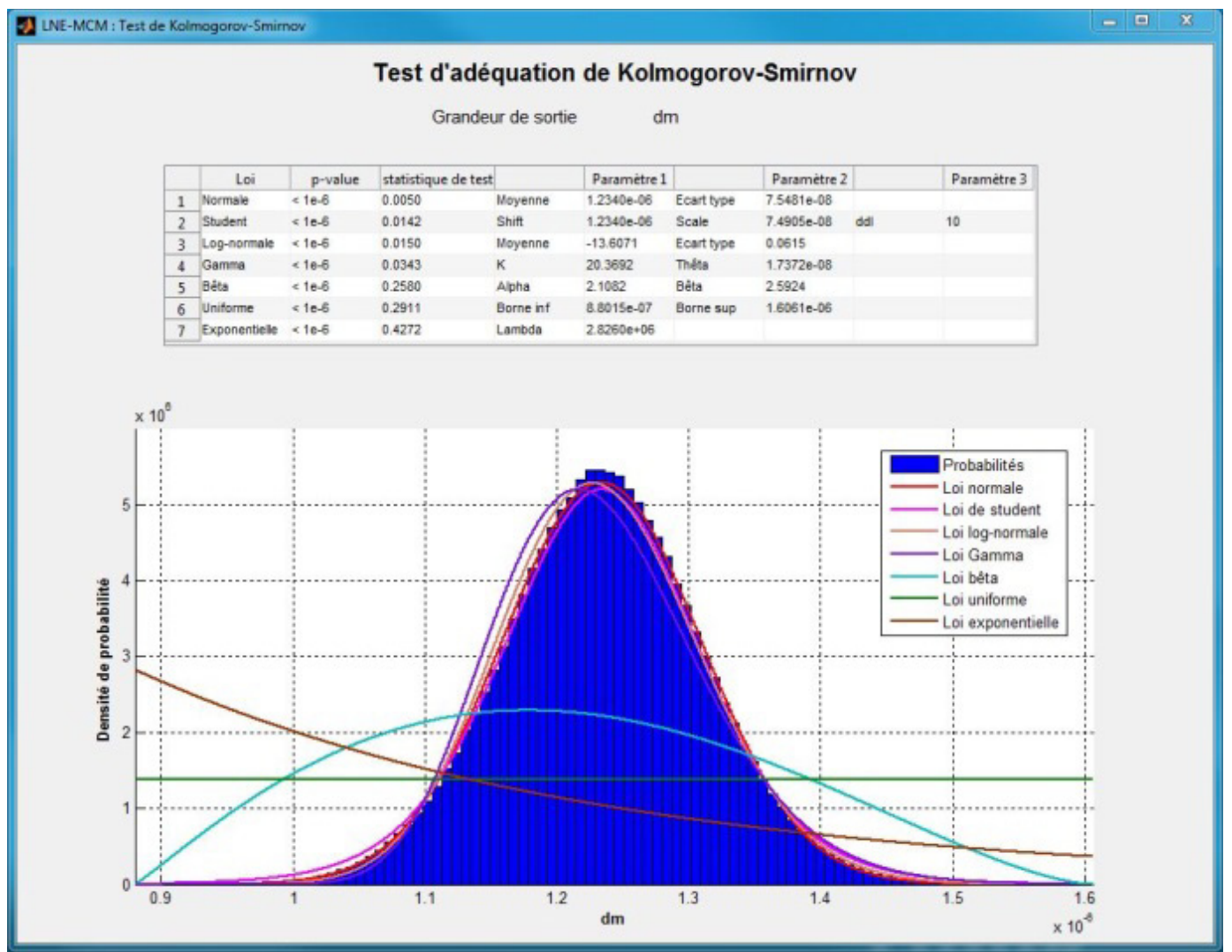

Fig. 6. - Test d'adéquation de la loi empirique de $d m$ par sept lois théoriques.



Fig. 7. - Analyse de sensibilité par la méthode des indices de Sobol'.

permet d'identifier facilement les contributions des différentes sources d'incertitude à l'incertitude finale (fig. 7), exprimées en termes de contribution à la variance de la grandeur de sortie. Le tableau accompagnant le camembert résume les valeurs des indices de Sobol' estimées par la méthode de Monte-Carlo.

Un autre exemple, traité dans le Supplément 2 du GUM, illustre l'analyse multivariée conduite dans le logiciel.

\subsection{Changement de repère (Supplément 2 du GUM)}

Les deux modèles mathématiques qui décrivent la conversion entre un système de coordonnées cartésiennes $\left(X_{1}, X_{2}\right)$ et un système de coordonnées polaires $\left(Y_{1}, Y_{2}\right)$ sont donnés par $[8, \S 9.3]$ :

$$
Y_{1}=\sqrt{X_{1}^{2}+X_{2}^{2}} \quad \text { et } \quad Y_{2}=\tan ^{-1} \frac{X_{2}}{X_{1}}
$$




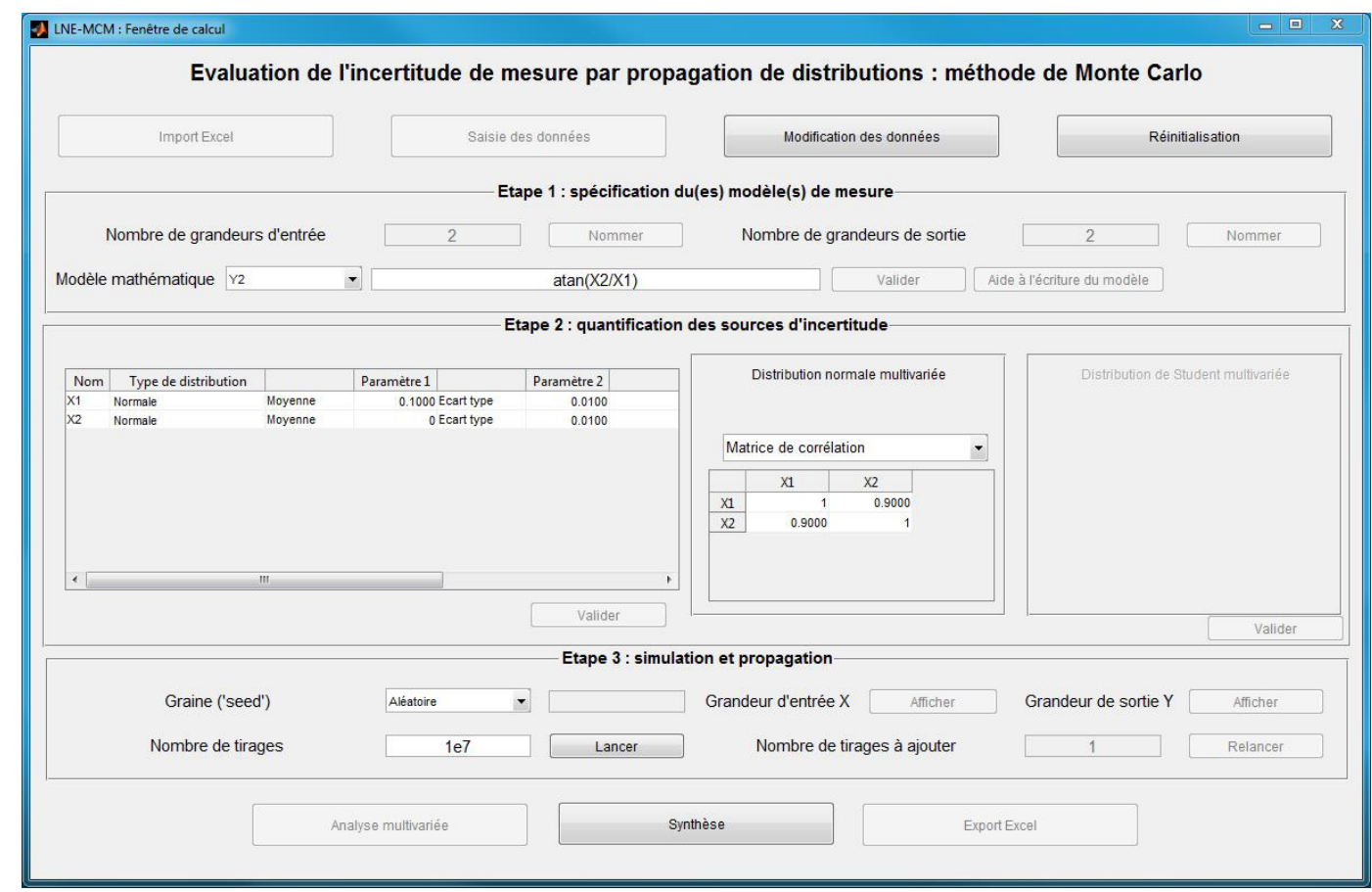

Fig. 8. - Fenêtre de calcul relative à l'exemple du changement de repère.

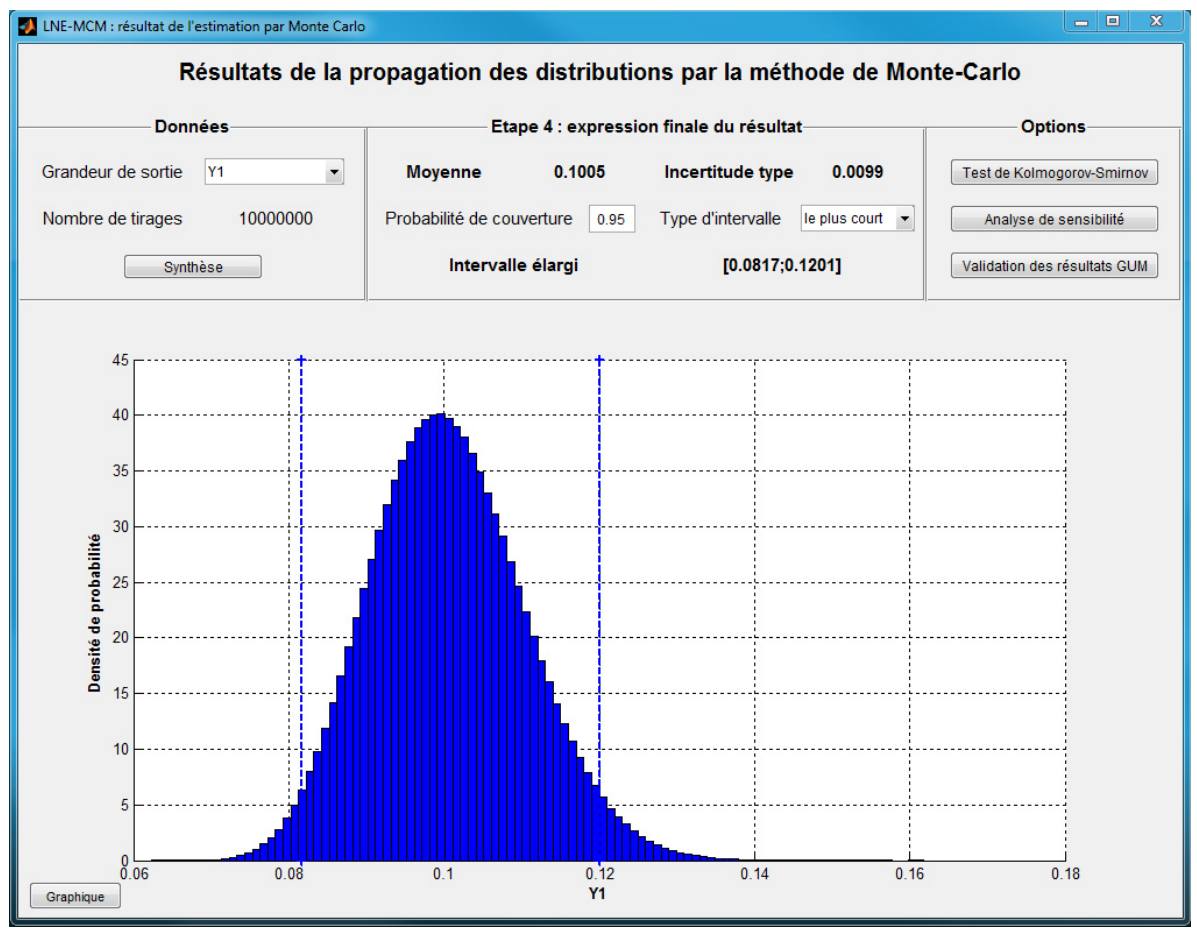

Fig. 9. - Résultats obtenus par la méthode de Monte-Carlo pour le module $Y_{1}$.

Les deux grandeurs d'entrée $\left(X_{1}, X_{2}\right)$ suivent respectivement une loi normale $N(0,1 ; 0,01)$ et $N(0 ; 0,01)$. On suppose qu'il existe un coefficient de corrélation $r=0,9$ entre les deux grandeurs d'entrée.

Les résultats concernant le processus de mesure sont renseignés dans la fenêtre de calcul du logiciel (fig. 8).
Lorsque les simulations de Monte-Carlo ont été effectuées, l'utilisateur obtient les résultats du calcul d'incertitude pour chacun des mesurandes (l'illustration sur la fig. 9 représente les résultats pour le mesurande $Y_{1}$ ).

L'analyse multivariée donne accès au coefficient de corrélation entre les estimations des deux mesurandes et ceci pour tous les mesurandes présents 




Fig. 10. - Analyse multivariée des mesurandes $Y_{1}$ et $Y_{2}$.

dans la modélisation mathématique. Le nuage de points que l'on observe sur la figure 10 représente les valeurs simulées des mesurandes spécifiés. La région élargie est matérialisée par une ellipse. Dans cet exemple, la distribution jointe représentant les évaluations des deux mesurandes $Y_{1}$ et $Y_{2}$ n'est pas gaussienne. L'ellipse n'est pas une bonne représentation de la région élargie d'aire minimale, puisqu'elle inclut des zones de plus faible probabilité (proche des limites du nuage de points bleus) et exclut des zones de plus forte probabilité (intérieur du nuage de points bleus).

\section{Conclusion}

Le logiciel LNE-MCM permet de mettre en œuvre la méthode de propagation des distributions par des simulations de type Monte-Carlo selon le GUM-S1. Ce logiciel, disponible gratuitement sur le site internet du LNE [4], a été conçu pour permettre d'évaluer l'incertitude de mesure selon la méthode de Monte-Carlo, de façon simple et intuitive.

Dans ce logiciel, d'autres fonctionnalités sont disponibles pour exploiter davantage les résultats obtenus afin de mieux connaître le processus de mesure étudié. En particulier, l'analyse de sensibilité permet d'identifier les principales sources d'incertitude associées à la grandeur de sortie. Le test d'adéquation permet de comparer l'échantillon simulé à une loi théorique connue, ce qui est une information importante pour utiliser la grandeur

Article reçu le 10 juin 2016, version révisée reçue le 7 novembre 2016. de sortie comme grandeur d'entrée d'un nouveau processus de mesure. Ce logiciel peut aussi être utilisé pour valider les hypothèses de la méthode du GUM (linéarité du modèle, loi gaussienne pour la grandeur de sortie). Il est également possible de prendre en compte le cas d'un mesurande multiple, sans considérer ce cas, comme souvent, comme des mesurandes indépendants.

\section{Références}

[1] ISO/CEI GUIDE 98-3:2008, "Uncertainty of measurement - Part 3: guide to the expression of uncertainty in measurement (GUM :1995)", 2008.

[2] Damasceno J.C et al., Estimation of primary $\mathrm{pH}$ measurement uncertainty using Monte Carlo simulation, Metrologia, 43, 3, 2006, DOI:10.1088/0026-1394/43/3/014.

[3] ISO/CEI GUIDE 98-3/S1:2008, "Uncertainty of measurement - Part 3: guide to the expression of uncertainty in measurement (GUM :1995), Supplement 1: propagation of distributions using a Monte Carlo method", 2008.

[4] Logiciel LNE-MCM, version 1.12015 , http://www.lne.fr/ fr/logiciels/MCM/logiciel-lne-mcm.asp.

[5] Matsumoto M. et Nishimura T., "Mersenne twister: A 623-dimensionally equidistributed uniform pseudorandom number generator", ACM. Trans. Model. Comput. Simul., 8, 3-30, 1998, DOI:10.1145/272991.272995.

[6] ISO 28640, "Random variate generation methods", 2010.

[7] Possolo A., "Copulas for uncertainty analysis", Metrologia, 47, 2010, 262-271, DOI:10.1088/0026-1394/47/3/017.

[8] ISO/CEI GUIDE 98-3/S2:2011, "Uncertainty of measurement - Part 3: guide to the expression of uncertainty in measurement (GUM : 1995), Supplement 2: extension any number of output quantities", 2011.

[9] SoвоL' I.M., "Sensitivity estimates for nonlinear mathematical models", Modelling and computational experiments, 1, 1993, 407-414.

[10] Allard A. et Fischer N., "Recommended tools for sensitivity analysis associated to the evaluation of measurement uncertainty", Advanced Mathematical and Computational tools in metrology and Testing IX, World Scientific, 84, 2012, 1-12.

[11] Massey F.J., "The Kolmogorov-Smirnov Test for Goodness of Fit”, J. Am. Statist. Assoc. 46, 1951, 68-78, DOI: $10.2307 / 2280095$.

[12] Saltelli A., Chan K. et ScotT E.M., "Sensitivity Analysis", Wiley, 2008, ISBN: 978-0-470-74382-9. 